\title{
El estilo de Max Weber. Sobre su participación en política y sobre el modo científico de escribir sociología
}

\author{
Álvaro Morcillo Laiz
}

\section{Resumen}

Las cartas escritas por Max Weber durante los dos últimos años y medio de su vida podría servir como base para una sociología de la intelligentsia centroeuropea de entreguerras. Sin embargo, en vez de centrarse en los encuentros de Weber con las figuras de su tiempo, tanto de la literatura - Thomas Mann, Hugo von Hofmannsthal - como de la música — Richard Strauss — , este artículo discute sucesivamente tres temas que relacionamos a menudo con Weber, pero que no son bien conocidos entre nosotros: primero, su participación, y la de alguno de sus estudiantes, como Georg Lukaçs, en contra o a favor de las revoluciones que siguen al final de la Primera Guerra Mundial; segundo, su estilo de escritura, a menudo denostado, pero que es su solución para un reto importante para su propia carrera como catedrático. Este reto, el tercer tema de este artículo, fue la constitución de la sociología académica como una disciplina académica que combinase el interés por el impacto de los hechos, del pasado y de las instituciones en la acción, como pretendía la Escuela Histórica de la Economía Política, con el uso de modelos, o tipos ideales, propugnados por la Escuela austriaca. A esta contribución intelectual, largamente olvidada, como intermediador entre la economía y la sociología, le corresponde una etapa biográfica, la breve estancia de Weber en la Universidad de Viena en 1918.

Palabras clave: Max Weber, clásicos de sociología, historia de las ciencias sociales, sociología económica, historia del pensamiento económico. 


\section{Abstract}

\section{Max Weber's style. On his involvement in politics and on the scientific way of writing sociology}

During the last two and a half years of his life, Max Weber wrote letters that could be used to write a sociology of the Central European intelligentsia during the wars. Rather than discussing Weber's encounters with the most renowned writers - Thomas Mann, Hugo von Hofmannsthal - and musicians — that of Richard Strauss, among several composers and conductors - of his time, this article discusses three more conventionally Weberian topics, which are nonetheless insufficiently understood: first Weber's involvement, that and of some of his students, like Georgy Lukaçs, in politics at the end of World War I. Second, Weber's writing style, which has been frequently decried, but that actually represents his attempt to overcome a challenge that had become important for his own project as a university professor. This is the third topic of the article: how Weber intended to found academic sociology as a discipline that should combine empirical facts, the historical past, and institutions, on the one hand, with the use of models, or ideal-types, as propounded by the Austrian School, on the other hand. To Weber's short spell at the University of Vienna in 1918 corresponds an intellectual achievement as mediator between economy and sociology that has rested too long in oblivion.

Kew words: Max Weber, classical sociology, history of social sciences, economic sociology, history of economic thought.

Dentro de las ciencias sociales, pocos autores son de tal envergadura como para justificar la edición de sus obras completas, sobre todo si la empresa se lleva a cabo con la ambición con que ha progresado la Max Weber Gesamtausgabe (MWG). ${ }^{1}$ Gracias a ésta, y a las voluminosas biografías aparecidas en los últimos años (Radkau, 2011; Kaube, 2014; Käsler, 2014), es difícil imaginar un científico social sobre cuya vida sepamos tanto como sobre Weber, con lo cual se abren vías para explorar la contingente historia de las ciencias sociales, así como la política, los intelectuales y la burguesía de un primer tercio del siglo XX, con el que seguimos conviviendo. A pesar de la cantidad de información ya disponible sobre Weber, las cartas son reveladoras en extremo y confirman lo que desde hace tiempo ha sido un lugar común

${ }^{1}$ Max Weber (2012), Briefe 1918-1920, en Gerd Krumeich y M. Rainer Lepsius (eds.), Max Weber Gesamtausgabe. II/10, Tubinga, J. C. B. Mohr (Paul Siebeck), lxii+1225 pp. 2 vols. Agradezco sus críticas y sugerencias a Eduardo Weisz, Esteban González y Alberto Penadés, así como al editor y los dictaminadores de Estudios Sociológicos. Asimismo, reconozco la ayuda editorial de Emilia Escamilla. 
entre los especialistas: la justificación de la MWG no es la edición y comentario de las publicaciones que Weber mandó a la imprenta sino las tres mil cartas que se han recuperado, así como los siete volúmenes de notas y apuntes de sus clases. En el caso concreto de las misivas que comento a continuación, su valor está en que deshacen lugares comunes, como el del descuidado estilo de escritura y el de que Weber fuera simplemente el profeta de la "sociología comprensiva" [verstehende Soziologie]. En realidad, le preocupaba escribir con precisión para que las ciencias sociales fueran rigurosas, para lo que debían no sólo reconstruir el sentido subjetivo de la acción, sino emplear modelos - tipos ideales_-, así como las matemáticas y la estadística.

La elevada cifra de cartas, más de 450, contenidas en estos dos volúmenes, se corresponde con el elevado número de destinatarios, más de 100, y con la enorme variedad de temáticas. Entre éstos encontramos a personajes que a los científicos-sociales contemporáneos y los historiadores interesan, como a Martin Buber, Paul Honigsheim, Karl Jaspers, Emil Lederer, Karl Loewenstein, Robert Michels, Otto Neurath, Gustav von Radbruch, Heinrich Rickert y Karl Vossler, además de otros a los que me referiré en este artículo. Aparte, en las cartas Weber menciona a políticos alemanes como Friedrich Ebert, Kurt Eisner, Walter Rathenau, Karl Liebknecht, Gustav Stresemann y Guillermo II, así como a otros extranjeros - George Lloyd y Woodrow Wilson-; a los austromarxistas Max Adler, Otto Bauer y Karl Kautsky; a colegas y estudiantes como Ernst Bloch, Siegmund Hellmann y Melchior Palyi; los editores de su Allgemeine Wirtschaftsgeschichte (Historia económica general), Hans Kelsen, Ludwig von Mises, Max Scheler, Carl Schmitt, Gustav von Schmoller, Georg Simmel, Werner Sombart, Rudolf Stammler, Ferdinand Tönnies, Ernst Troeltsch, y su hermano Alfred; y a literatos como Stefan George, el conde Hermann von Keyserling, Rainer Maria Rilke, Oswald Spengler; además de a distintas autoridades universitarias y redacciones editorial y periodísticas. Las misivas han llegado a nosotros gracias a que la esposa de Weber, Marianne, conservó prácticamente todas las que le envió, al igual que la editorial de Max, J. C. B. Mohr (Paul Siebeck); pero también por las que de terceros recibió Eduard Baumgarten, el sobrino-segundo de Weber, así como de varios archivos públicos y de colecciones privadas. Siguiendo las ambiciosas normas de la MWG, cada una de las cartas ha sido editada de modo que ha sido datada, si el original no lo indicaba, el lugar en que se escribió indicado, el contexto de la misma explicado en una nota que la precede y se ha ofrecido información sobre aspectos concretos mediante notas al pie. Cada documento está editado de modo que puede leerse de forma independiente, sin necesidad de consultar los anteriores. Ello es posible, en gran parte, porque las cartas van acompañadas de índices — cronológico 
de cada una, de las personas mencionadas por Weber, de todas las personas a las que se alude en la publicación y de lugares-. El resultado es una edición un tanto aparatosa y un texto con reiteraciones, pero con una información útil $\mathrm{y}$ tan accesible que permite usar los dos volúmenes como si fuera una obra de consulta.

Los membretes muestran que Weber escribió estas cartas en Heidelberg, pero también desde Viena y Munich, donde ocupó sendas cátedras. En ellas habla de política y de ciencia social; también, por alusión, de la relación de la burguesía centroeuropea con la cultura; y de amor: a Marianne, pero también a Mina Tobler y sobre todo a Else Jaffé, un romance del que Joachim Radkau ha informado hasta la saciedad (Radkau, 2011; Morcillo Laiz, 2013). Dado que existe este título en español, así como otro en alemán sobre la familia de Max Weber (Roth, 2001), y que esta revista es sociológica, me centro aquí en los temas de interés más general para científicos sociales, dejando de lado otros que han quedado reflejados en estos volúmenes, como la participación de Weber en la política universitaria mediante dictámenes y otros escritos. Precisamente por su interés (Weber, 1974; Monroy, 2008), el tema habrá de ser discutido cuando los textos al respecto de Weber sean publicados (2015).

No hay espacio aquí más que para indicar que en las cartas Weber "reseña" los conciertos que escucha en Munich y Viena - Johannes Brahms, Josef Haydn, Franz Liszt, Richard Strauss - o se refiere a sus lecturas de Henrik Ibsen. Por supuesto, en las cartas a Else aparecen Lear, Shylock, Titania y el soneto 102 de Shakespeare, que Weber también cita al final de $L a$ política como vocación. En otras ocasiones, son los encuentros personales los que dan pie a esta crónica cultural: "Ayer estuve en casa del embajador sajón (von Nostiz) con Hugo von Hofmannsthal, quien decepciona un poco: un vienés inteligente, refinado, pero de lejos no tan refinadamente cultivado como "Tod des Tizians [La muerte de Ticiano] hace suponer" (a Marianne Weber, 17-06-1998: 199). ${ }^{2}$ Weber coincide con Thomas Mann en casa de amigos comunes muniqueses. Después de una de esas reuniones, el autor de Der Zauberberg [La montaña mágica], escribió en su diario que el profesor "polemizó en contra de Spengler y demostró ser el buen, habilidoso y vivo orador por el que se le tiene" (Mann, 1979: 352). Ese estilo al que alude Mann, al que volveré más tarde, se deja adivinar en las cartas en las que aparecen nuevas formulaciones, brillantes y claras, de ideas fundamentales de Weber: "Por supuesto que tenemos ante nosotros personas que valoran co-

\footnotetext{
${ }^{2}$ Salvo que se indique algo en contrario, todas las referencias son a cartas contenidas en MWG II/10 y se indica el destinatario, la fecha y la página en que se encuentra.
} 
mo objeto de observación, pero nosotros no estamos obligados a valorar" (a Rickert, 26-04-1920: 1040-1041).

A lo largo de más de mil páginas atravesamos episodios cruciales, no sólo de la vida y la obra de Weber sino de las primeras décadas de un siglo XX europeo. Siendo imprescindible elegir algunos de esos episodios, este artículo toma tres como pretexto: el primero, al que dedico menos espacio pues es más conocido, es la participación política de Weber y de su entorno durante la primera posguerra. El segundo es su regreso a la docencia después de casi dos décadas, que le lleva a reflexionar en sus cartas sobre el problema de cómo escribir y enseñar sociología. Se ha divagado tanto sobre el mal estilo de Weber, sin llegar a entender que el modo de escribir de Weber es su respuesta al problema de cómo practicar y enseñar una sociología basada en conceptos y hechos. El tercer episodio concierne al paso de Weber por las facultades de economía de Munich y Viena, una institución esta última que resulta crucial para la historia del pensamiento económico europeo, como vínculo metodológico entre la sociología académica, que estaba por desprenderse de la Nationalökonomie [Política Económica].

\section{Los académicos y la política en la primera posguerra}

Entre los temas de relevancia política tratados en las cartas están el final de la primera guerra mundial y la negociación de la paz, incluido el Tratado de Versalles, a donde Weber viajó a regañadientes como parte de la delegación alemana, y las revoluciones de izquierdas en la inmediata posguerra: los espartaquistas, la "República bávara de los soviets". Entre las secuelas de aquel "carnaval sangriento" están también el testimonio de Weber en defensa de algunos jóvenes amigos y conocidos que habían jugado un papel destacado en la misma, como Ernst Toller, a quien Weber defiende ante un tribunal argumentando que el acusado no era un oportunista sino que se había dejado llevar por su "ética de convicción" [Gesinnungsethik]. También sus estudiantes participan en empresas políticas de izquierda, como Georg Lukaçs en la República Soviética Húngara de Bela Kun, quien después de un tiempo desaparecido acabó exiliado en Alemania; una carta de Weber al padre y otra al hijo dan cuenta del episodio:

Querido amigo, estoy contento de finalmente poder oír algo auténtico sobre su situación [Ergehen], pues espero no malentender al Sr. Dr. Mannheim. Desde entonces no supe nada más que mediante una carta muy desesperada de su señor padre y después ya nada más, salvo que está enredado en política o en sus consecuencias. 
Estimadísimo amigo, por supuesto que nos separan las opiniones políticas. [...] Sólo que cuando pienso lo que la agitación [Treiben] política actual (desde 1918) nos ha "costado", sin distinción de la "orientación", en valores indudables, por ejemplo Schumpeter, ahora, usted, y lo que aún nos ha de costar sin que, estoy convencido de ello, nos vaya a rendir siquiera lo más mínimo —itodos nos encontramos bajo una dominación extranjera!-, siento un poco de amargura respecto a este absurdo destino. [...] Y de esa atmósfera vienesa, de ahí tiene que marcharse. Allí todo se mueve en círculos. A Italia o a Alemania, puesto que París ahora me lo imagino imposible. [...] De todos modos, este asunto tiene que poder arreglarse de algún modo y usted, ahora que todo se volverá reaccionario por décadas, ser devuelto a las tareas que usted mismo y sus facultades le han impuesto. (A Lukaçs, febrero o marzo de 1920: 961-962)

En esos mismos años, Weber, por su parte, colabora activamente con el liberalismo, en el Deutsche Demokratische Partei. Si bien fracasa en el intento de obtener un escaño en el primer parlamento alemán de la posguerra, compensa la desventura con la actividad periodística para el Frankfurter Allgemeine, desde donde sus editoriales y artículos, sobre todo "Parlamento y gobierno en una Alemania reorganizada", tienen considerable repercusión. En parte debido a éste, Weber es llamado, en medio de los diversos intentos revolucionarios, a colaborar en la redacción de la que será la constitución de la República de Weimar, a la que Weber hizo una pequeña, pero no despreciable contribución. Sí fueron incorporadas sus sugerencias sobre las comisiones de investigación parlamentarias, las constituciones de las entidades federales y la autonomía de la administración local. Por el contrario, la propuesta a favor de la elección directa del presidente [plebiszitäre Führerdemokratie] tuvo menos impacto sobre los distintos proyectos constitucionales, pero más en la opinión pública alemana del momento. El respaldo de Weber a un líder con poderes amplios y un fuerte respaldo en las masas, que sirviera al presidente para enfrentarse al parlamento y a los partidos, fue lo que dio pie a que se arguyera que había sido un profeta del autoritarismo. Esta tesis, que Wolfgang Mommsen (2004; 1984) publicó por primera vez en 1959, abonaba a su vez el terreno a quienes desde la teoría crítica rechazaban a Weber, hizo peligrar la recepción de su obra en Estados Unidos y Alemania, por lo que Reinhard Bendix y Talcott Parsons tuvieron que tratar de contrarrestarla, poniendo el énfasis en un Weber liberal (Stammer, 1965; 1971; Roth, 2006). Las actividades políticas, y sus rastros en las cartas, constituyen el contexto en que han de entenderse las conferencias sobre la vocación, en especial Politik als Beruf.

Después de apartarse de la convulsa política de la posguerra Weber se adentra en una fase de intensa actividad intelectual, que se intensifica a partir 
de mediados de 1919. Es más, inicia el año crucial en que trata, sin conseguirlo, de culminar sus dos grandes obras, La ética económica de la religiones universales y Economía y sociedad. Dado que Weber murió repentinamente en junio de 1920, dejando sólo parte de estas dos obras en la imprenta, las cartas de ese año y de los dos anteriores son una de las fuentes principales para entender qué pretendía hacer con las galeras de Economía y sociedad, que no terminó de revisar, y con los otros manuscritos que tal vez estaban destinados a ser parte de la misma.

La luz que ilumina estos problemas proviene casi siempre de las cartas presentadas, hasta ahora disponibles sólo para la media docena de especialistas que saben descifrar la letra de Weber. En ellas se basa el artículo de Mommsen aparecido recientemente en Estudios Sociológicos (2014). La relevancia de la publicación de las cartas como parte de la MWG reside en que aportan una fuente fundamental para entender un episodio editorial crucial para la historia de las ciencias sociales en el siglo XX. En esta correspondencia entre Weber y el editor, y entre Marianne Weber y los sucesores de dicho editor, se basa la serie de artículos aparecidos entre finales de los años setenta y de los noventa que han llevado a la "deconstrucción" de Economía y sociedad, incluyendo piezas cruciales como Abschied von Wirtschaft und Gesellschaft [Despedida de Economía y sociedad] de Friedrich Tenbruck (1977) y Das Ende eines Mythos [El final de un mito] de Wolfgang Schluchter (1989). Desde entonces no cabe ya duda de que antes de morir Weber había descartado gran parte de lo que la segunda parte de Economía y sociedad contiene hoy en día.

\section{Descuidar la escritura para favorecer a la sociología}

Esta correspondencia es notable no sólo por lo que en ellas Weber afirma sobre el final de la guerra o la política en la futura Alemania, sino por cómo se expresa en ellas. El estilo de Weber ha sido muy discutido, casi siempre para criticarlo. En el prefacio a una de las traducciones más leídas de Weber, Hans Gerth y C. Wright Mills (1946: v) asignan a su autor a una tradición de escritura que no se dirige al oído, como Friedrich Nietzsche o Franz Kafka, sino al ojo del lector. Autores en esta tradición, "use parentheses, qualifying clauses, inversions, and complex rhythmic devices in their polyphonous sentences. [...] At their best, they erect a grammatical artifice in which mental balconies and watch towers, as well as bridges and recesses, decorate the main structure".

Por el contrario, el editor de la traducción de Economía y sociedad al inglés afirma que "Weber wrote lucidly and subtly. He wrote more clearly 
than did most of his colleagues, including Sombart, Tönnies, Troeltsch and his own brother, Alfred" (Roth, 1978: cvii).

A pesar de que textos de Weber como las ya mencionadas conferencias y el final de La ética protestante son obras maestras de la retórica, pueden nombrarse al menos tres razones por las que el estilo de Weber ha sido a menudo difamado. Una de ellas es que la inmensa mayoría de sus críticos no se refieren al alemán de Weber sino a traducciones que a menudo han convertido originales difíciles, pero precisos, en traducciones vagas y apenas comprensibles (Morcillo Laiz, 2014). Otra es que algunos de sus textos más conocidos, como la segunda parte de Economía y sociedad, nunca fueron enviados por Weber a la imprenta sino que lo hizo Marianne. Responsabilizarle por sus borradores no ayuda a entender por qué los textos cuya publicación sí decidió estaban escritos de un modo y no de otro. Sin embargo, es absolutamente cierto que Weber publicó textos que siguen pidiendo a gritos un editor. Al respecto, es famosa la crítica, muy justa, de Hermann Kantorowicz al ensayo de las categorías a la que el autor de Economía y sociedad responde: “Sociología comprensiva' — ¿Incomprensible? ¿Para usted? — 'Pues si esto sucede con la madera verde' ['¿qué pasará con la seca?’, Lucas, 23: 31; ÁML], —CCuán miserablemente debo haber redactado!" (Weber, 1985a; 1973a; 2003: 442). Tan justificada es la crítica que los aspectos formales del artículo, que abre con varias polémicas de Weber contra la psicología y el derecho y hoy sólo de interés para la historia de la ideas, han impedido hasta hoy que se aprecie la importancia de las páginas finales, que contienen in nuce una teoría del Estado y de la racionalización. Finalmente, en otros casos, como veremos, el estilo difícil es el tributo que Weber acepta pagar en aras de una precisión que consideraba imprescindible para la nueva sociología que él propugnaba.

El propio Weber tematiza el dilema del estilo en algunas de sus publicaciones. Así, muestra ser consciente de las dificultades que plantea "la pedante minuciosidad de las formulaciones" (Weber, 1973a: 175, n. 1.; 1985a: 427, n. 1), y de la necesidad de justificarla. Así, al inicio de Economía y sociedad escribe que "el deseo de una popularización incondicionada no sería siempre compatible con el rigor conceptual y debe ceder ante éste..." (1944: I:3; 1922: 1). La escritura pedante pero precisa con que trató de superar este reto difiere del tratamiento conceptual "fluido, proteico" que favorecen los sociólogos (Breuer, 2014). Esa diferencia hace aún más bienvenidas las observaciones sobre el problema de la expresión escrita que encontramos en estas cartas, en las que una y otra vez Weber compara el estilo en que se escribe y el que emplea al dar clases. Alguien que afirma "Lo que pasa es que soy un hombre de la pluma, no de la cátedra" (a Marianne Weber, 28-6-1919: 667), observa también que "Lo 'cansado' en las lecciones está — ahora me doy cuenta- 
en que, en mi caso, el estilo de hablar y el de escribir son completamente distintos. Sin cortapisas puedo hablar sólo en un discurso libre (siguiendo apuntes). En clase debo ofrecer formulaciones 'responsables' y ello produce un agotamiento absurdo, a mí al menos" (a Mina Tobler, 26-07-1919: 704). Lo anterior puede parecer propio de un neurasténico, pero cuando uno piensa en el grado de abstracción y la densidad de las primeras páginas de Economía y sociedad, que contienen su Conceptos fundamentales queda clara la dificultad, y lo agotador, de dar una clase a cientos de estudiantes. Precisamente eso es lo que Weber estaba tratando de hacer en aquellos años.

Las cartas contienen numerosas observaciones como las apenas citadas sobre el estilo con que da clases. Esta obsesión difícilmente puede sorprendernos, dado que sabemos que las dificultades con la docencia contribuyeron, al parecer, a la crisis nerviosa de 1898 que le mantuvo alejado de las aulas por casi veinte años. Más llamativa pudiera aparecer la presencia continua de su vocabulario científico en cartas personales. Al leerlas, encontramos conceptos de Weber que conocemos de sus publicaciones: racionalidad, mecanización, diletantismo, burocracia, etc.; como otros científicos sociales, Weber estaba intentando entender su mundo; y a sí mismo (Hennis, 1983; 1987a; 1987b; 2015). A su hermana, Lili Schäfer, le escribe sobre sus superiores en la Odenwaldschule Oberhambach, que "no son tal vez suficientemente responsables como para soportar la racionalización completa de la explotación" (23-09-1919: 788-789). Para honrar la memoria de Friedrich Naumann, su mentor liberal en la política, afirma que éste era alguien "a quien la política no le dejaba vacío como persona, no lo mecanizaba o lo hacía brutal o refinado..." (a Magdalene Naumann 27-08-1919: 742). A su amiga y pianista, Mina Tobler, le explica desde el Munich posrevolucionario que "Este socialismo 'diletante' es un tipo desesperante de burocracia. Lo que antes necesitaba veinticuatro horas, requiere ahora ocho días" (04-09-1919: 755756). Weber hace un uso inesperado, y de dudoso gusto, de la teoría de la acción, cuando le cuenta a Tobler: "Hacia la seguridad de tu 'estilo' y el nivel de tu conducción de vida se orienta mi propio ser" (12-06-1919: 644). Poco después escribe a su amada Else: "Todo, todo en mí se 'orienta' a tu voluntad". Y a continuación admite: "Como ves, cito de las 'clases' [Kolleg]" (26-08-1919: 740). El prurito por la precisión lleva a Weber a acudir a sus "conceptos fundamentales" en sus cartas personales.

La lectura de la correspondencia muestra que Weber puede emplear oraciones decididamente breves, sin apenas subordinadas; las frases tal vez no sean sutiles pero sí están llenas de alusiones, sin dejar de ser directas y lúcidas. A pesar de las diferencias en la escritura, el estilo intelectual de Weber es reconocible en las cartas, pues se caracterizan, al igual que sus 
mejores textos académicos, por los mensajes agudos, precisos, combinados con puntualizaciones; un manierismo producto del esfuerzo por combinar generalizaciones históricas con causas específicas (Roth, 1978: cviii). Al igual que en sus publicaciones, Weber recurre en sus misivas al mismo uso profuso de subrayados y comillas que caracteriza sus ensayos, que tantos problemas ha causado a editores y traductores (Morcillo Laiz, 2012: 618-620), pero que refleja su obsesión con la adecuación terminológica. Llevando al extremo estas reflexiones: si Weber redactaba "mal" es por decisión propia.

\section{Qué tomar de la economía política para dárselo a la sociología}

La presencia de los términos de su "sociología" en el lenguaje cotidiano parece una consecuencia de la obsesión de Weber por los conceptos. Ésta no es un capricho sino, en su opinión, una respuesta a las que él cree que son las necesidades imperiosas de la disciplina. Precisamente en los años que preceden a su muerte, Weber trata de encontrar un lugar desde el cual poder investigar la influencia en la vida económica de los aspectos sociales o, en su vocabulario, de los órdenes [Ordnungen] y, en la jerga de hoy, institutions. Dentro una disciplina mucho más amplia de lo que hoy entendemos por economía, la Sozialökonomik, Weber trata de encontrar un lugar, junto a la teoría y la historia económicas, para la sociología. La opinión que tenía Weber de lo que se ofrecía en la universidad y fuera de ella bajo esta etiqueta no podía ser peor: "[Franz] Oppenheimer tiende precisamente a dibujar amplias panorámicas precisamente sin aquello que a una joven disciplina como la sociología le hace más falta: el respeto incondicional a los hechos concretos, por un lado, y la delimitación exacta de los conceptos, por el otro" (a colega desconocido, 15-12-1918: 368). El vínculo entre una disciplina que carece de profesionales formados en la misma, y que descansa en manos de quienes se han sentido atraídos hacia su órbita, se plasma en los deseos de Weber de que "la 'sociología', cuya práctica la universidad no tendrá más remedio que atender, no caiga en manos de diletantes, sino [...] en las mías" (a Walther Lotz, 21-02-1919: 472).

No sólo en la docencia trata Weber de solucionar el problema de los conceptos, también en sus textos. Sobre Economía y sociedad escribe a su editor: "la versión existente debe ser revisada con precisión, precisamente porque le estoy dando la forma 'didáctica', que considero apropiada para finalmente tratar la 'sociología' de modo rigurosamente especializado y científico, en lugar de las contribuciones propias de diletante por filósofos ocurrentes" (a Paul Siebeck, 08-11-1919: 833). Además de Oppenheimer, 
Weber tiene en mente autores como Oswald Spengler, en cuyo libro ve, a pesar de "las numerosas ocurrencias y de que la ciencia, al igual que el arte, necesita la compañía del diletantismo", el riesgo de que "un literato así le arruine a la gente el gusto por el trabajo sobrio y el respeto ante los hechos" (a Mina Tobler, 03-01-1920: 877). Las consideraciones metodológicas y el peligro que constituyen los aficionados para la institucionalización de la sociología son sólo parte de las preocupaciones de Weber.

En realidad, su proyecto para la disciplina va más allá de la definición de conceptos. Como veremos, Weber trata, por un lado, de construir teoría y, por el otro, estudiar "los órdenes y poderes sociales", es decir, las instituciones concretas que afectan a la vida económica y al hombre moderno. Al hacer suya esta pregunta, Weber se coloca en el margen de la Nationalökonomie, la disciplina cercana a la economía política dentro de la que se había desarrollado su carrera académica, pero muy próximo a la sociología, una actividad intelectual en aquel momento poco respetable para la que prácticamente no existían cátedras en la universidad alemana. El que Weber nunca ocupara una de ellas es poco sabido, a pesar de los trabajos sobre las relaciones entre la sociología y la economía en aquel periodo que han publicado Keith Tribe $(1995 ; 2012 ; 2015)$ y Richard Swedberg $(1998 ; 2002 ; 2007)$. Menos aún se entiende la importancia de los vínculos con la Escuela Austriaca para establecer la especificidad de la propuesta de Weber para las ciencias sociales, una de las cuestiones que las cartas ayudan a captar. Ese malentendido abarca el estilo porque en él se refleja que con su parole hace un Spagat —abre las piernas - abarcando dos langues rivales y opuestas (Pocock, 1987: 20), la de la Escuela Histórica y la de la Austriaca, lo cual hacía más difícil entender a Weber que a un autor que está firmemente anclado a una sola tradición y su langue.

Como hemos visto, los conceptos "bien definidos y unívocos" son parte del esfuerzo de Weber por legitimar $s u$ sociología frente a sus propios colegas. A pesar de que trabaja en una facultad de economía o, más bien en una Staatswirtschaftliche Fakultät [Facultad de economía del Estado], en la que ocupa la cátedra que fue de una economista (Lujo Brentano], Weber parece a veces que se sienta a justificarse. El tono de una larga misiva a Robert Liefmann es apologético; en ella interpela al destinatario, un teórico, si bien uno más "conservador" que los de la Escuela Austriaca. En defensa de la economía como una disciplina que abarcase mucho más de lo que hoy se considera tal, Weber le espeta: "Puede entenderse con Schumpeter mucho mejor de lo que usted mismo cree" (09-03-1920: 948). Es revelador que Weber ensalce al autor de Theorie der wirtschaftlichen Entwicklung (Schumpeter, 1944; 1912) — “Schumpeter es el más capaz” (a Paul Siebeck, 16-02-1802: 
90)— porque éste, a pesar del éxito de sus trabajos en la segunda década del siglo XX, aún no es una figura consagrada como von Wieser. De hecho, Weber aún esperaba ver a Schumpeter, cuya cátedra estaba en ese momento en Graz, ascender hasta una de las grandes universidades germánicas: "Schumpeter debería sin duda irse a Berlín. Desde allí le sigue estando abierto todo. Por el momento no se puede hacer nada por él" (a Emil Lederer, 03-03-1920: 937). Weber ya había fomentado anteriormente tal ascenso mediante una serie de dictámenes emitidos con motivo del nombramiento de un sucesor para la cátedra de Friedrich von Wieser.

Conviene citar in extenso otra carta anterior en la que se pone de manifiesto la combinación de virtudes que según Weber debía reunir en aquel momento un catedrático de economía. El parecer compara a dos candidatos, Schumpeter y el primer gran estadístico moderno en Alemania, Ladislau von Bortkiewicz:

Ambos sin duda estudiosos de primera categoría, lo cual no puede decirse de otros, cualesquiera que sean sus demás méritos (es decir, no lo son en el campo de la teoría económica). El profesor von Bortkiewicz [...] como estadístico no ha sido igualado, bien entrenado en todos los aspectos de la teoría, en esto tal vez se ha destacado más como crítico que como proponente y creador [...] A este respecto, Joseph Schumpeter seguramente le supera [como docente, ÁML], al igual que en todo aquello que ofrece un intelecto rico, más joven, que aspira aún a la plenitud y a expresarse. En cuanto a la producción ha aportado creativamente más - claro que hasta ahora también más desaciertos- que el profesor von Bortkiewicz, que está por encima de él en cuanto a serena seguridad y además como estadístico. [...] Personalmente esperaría de él [Schumpeter] como estudioso, en especial como teórico, algo grande, si se integra a tiempo en un ámbito de actuación adecuado. En sus fascinantes dotes como docente nos supera, creo yo, a todos nosotros y no se puede negar que continuará la vieja tradición vienesa de manera muy brillante. (A Carl Grünberg, 04-06-1918: 176-178)

Los temores implícitos de Weber a que Schumpeter arruinase su carrera no estaban del todo infundados, pues después de la guerra su joven colega se lanzó primero a la política austriaca como ministro de hacienda y luego a la banca. La primera aventura acabó en acusaciones de haber especulado, concretamente mediante agiotaje, y la segunda en la asunción de deudas a título privado (Swedberg, 2001: 13600). La participación de Schumpeter en el gobierno de Karl Renner en 1919 impidió que se le ofreciese una cátedra en Munich:

Schumpeter no ha sido posible no por causa de la "política" en sí sino por su comportamiento totalmente voluble [...]. Lo he defendido en todas las circuns- 
tancias $[\ldots]$ pero no se podía hacer nada. En el futuro también hablaré a su favor y considero que el no darle el nombramiento es una desgracia para nosotros. Pero no era posible. ¿Qué tiene que hacer alguien así en la política? Una persona debe saber lo que no puede. (A Emil Lederer, 16-02-1919: 918-919)

En el pensamiento de Weber el estatus central de la investigación empírica $y$ de los conceptos lo sitúa entre la economía alemana y la austriaca. Puesto de otro modo, Weber, quien de joven se declaró uno de "los discípulos de la Escuela Histórica" (1988: 16; 1991), estaba entre quienes tenían un verdadero interés en el vínculo entre historia e instituciones económicas, como él mismo, y los teóricos económicos, cuya metodología consideraba acertada. Pero, ¿qué necesitaba Weber de la economía austriaca para su proyecto de una Sozialökonomik? Frente a la acumulación de datos y narraciones de la Escuela Histórica, Weber reconocía la importancia de la teoría económica y estaba a favor del uso de "modelos" así como del lenguaje matemático y de la estadística: "Supuestamente afirmo que el valor cognoscitivo de la teoría es 'reducido'. ¿Dónde pasaría tal cosa? La teoría crea tipos ideales y precisamente para mí este resultado es el más imprescindible" (a Robert Liefmann, 09-03-1920: 949). Es más, el tipo ideal, contra cuya proclamación como utopía nos alerta Weber (1985b: 534; 1973b: 263), parecería estar construido a semejanza de los conceptos económicos, como el de utilidad marginal, por lo que pueda afirmarse que Weber adopta la postura de Carl Menger, no la de su maestro Schmoller, en el Methodenstreit: "Weber, in his discussion of the nature and general function of concepts, sides with the abstract school and against the historical one" (Bruun, 1972: 209). Desde los años veinte, Ludwig von Mises, Friedrich von Hayek y otros autores vinculados a la Escuela Austriaca, como Alfred Schütz, discutieron la compatibilidad entre el concepto de tipo ideal en Weber y el de utilidad marginal (Swedberg, 1998: 204-205, 302-303).

Otro elemento distintivo de la propuesta de Weber para las ciencias sociales que le vincula con Schumpeter y la Escuela Austriaca es el énfasis en las acciones de las personas, el llamado methodologischer Individualismus, que, usando otros términos, Weber propone en el artículo programático para el primer número del Archiv für Sozialpolitik und Sozialwissenschaft (Weber, 1985c; Weber, 1973c), un énfasis que Schumpeter retoma en Das Wesen und der Hauptinhalt der theoretischen Nationalökonomie (1908). Parte del mismo es la necesidad de llevar a cabo un estudio subjetivo de la acción para entender las instituciones económicas. Igual de importante es el elemento histórico: si bien el objeto de estudio es una acción delimitada en el tiempo, la comprensión cabal de la misma exige atención a su historicidad y su compo- 
nente subjetivo. Convertir en aceptable en el mainstream sociológico y científico-político el estudiar la acción desvinculada del tiempo - y de los órdenes o instituciones - ha sido una de las malhadadas consecuencias de la recepción parsoniana de Weber (Zaret, 1980):

Lo determinante para la teoría es, por supuesto, el hecho de que sea percibido subjetivamente por el sujeto económico, la teoría es indiferente a la ética. -El estado de necesidades del individuo es histórico e individual cambiante y capaz de desarrollarse, y además esto último tanto en lo cuantitativo- ello en medida limitada — como en lo cualitativo- a este respecto, en principio, ilimitadamente. Toda la historia económica de Occidente es historia de la expansión cualitativa del estado de las necesidades. (Weber, 2009: 123)

La concepción de la acción como subjetiva, constitutiva de las instituciones [Ordnungen] y cambiante a lo largo del tiempo, establece el vínculo entre teoría e historia económica que en última década del siglo XIX une el trabajo de Weber y de Alfred Marshall (Tribe, 2012: 288). Dado que cada momento histórico es "único", si la historia determina la acción económica, entonces la acción económica no es reducible, en última instancia, a un modelo universal. Por consecuencia, el estudio empírico e histórico de la acción económica es imprescindible. Según Tribe (2012: 289), en los años treinta del pasado siglo el estudio de dicho vínculo habría ya quedado abandonado en beneficio de una concepción más formalizada de la "economía moderna", que volvió prescindibles a la sociología y la historia económicas.

Desde el punto de vista subjetivo propugnado por Weber, pueden estudiarse las acciones, incluidas las económicas; si bien para él éstas sólo son explicables del todo desde una perspectiva histórica, lo que le acercaba a la tradición en la que se había formado, la de la Escuela Histórica. Podría decirse que la tensión entre la importancia que da tanto a lo individual, histórico, subjetivo, por un lado, y a lo general que define los modelos o tipos ideales, por el otro, contribuye a la dificultad de su estilo.

Gran parte de estos contrastes se ponen de manifiesto en la citada carta a Liefmann, en la que, además, Weber ofrece la versión más concisa imaginable de su sociología del Estado:

Primero, su lucha contra la "sociología", que yo entiendo, pero quiero señalar que si yo ahora me he convertido en sociólogo (¡según mi contrato de trabajo!), ello se debe fundamentalmente a que quiero acabar con esa actividad [Betrieb] que trabaja con conceptos colectivos y que sigue creando fantasmas. En otras palabras, también la sociología puede hacer sólo si se parte del actuar de individuos, de uno, de pocos o de muchos, es decir, estrictamente "individualista" 
en el método. Sobre el "Estado" proclama usted, por poner un ejemplo, puntos de vista completamente anticuados. Desde el punto de vista de la sociología, el Estado no es nada más que la probabilidad de que determinados tipos de un actuar específico tengan lugar, actuar de determinados personas individuales. Nada más que esto. Esto es lo que escribo y enseño desde hace años. En ello lo "subjetivo" es que este actuar se orienta a ciertas representaciones. Lo "objetivo", que nosotros, los observadores, juzgamos si existe la probabilidad de que se lleve a cabo ese actuar orientado a representaciones. Si ya no existe, tampoco existe ya el "Estado" [...] Puesto que esto es cierto: como dije, la concepción cuantitativa, por ejemplo Schumpeter etc., que es inobjetable si se la entiende bien, puede llevar a consecuencias completamente equivocadas, si el teórico no presta metódicamente atención una y otra vez al carácter subjetivo — condicionado subjetivamente - de las acciones económicas. Pero si lo hace, la pregunta se vuelve en realidad meramente terminológica. Por el momento me dedico sólo a la sociología económica [Wirtschafts-Soziologie], a pesar de ello me vienen estupendas las construcciones bien definidas y, por lo que puedo ver hasta ahora, consecuentes. (09-03-1920: 954)

Las cartas relativas a Oppenheimer, Schumpeter o Liefmann contenidas en estos dos volúmenes nos ofrecen una perspectiva más rica sobre una de las posturas de Weber que le diferencian de su generación y le proyectan hacia el presente: tenía una idea de la ciencia social como un saber probabilístico en la que modelos - tipos ideales - comparables al del homo economicus se combinan con un conocimiento estrictamente empírico, el cual abarca un elemento hermenéutico desplegado para reconstruir la intención de los actores. En vista de estas ambiciones, el estilo de Weber constituye un intento de responder a preguntas relevantes con precisión conceptual. Incluso sus detractores deberían poder admitir que los problemas que se plantean los "clásicos", no necesariamente sus soluciones, son lo que debemos retener. Las cartas escritas durante los últimos años de su vida ayudan a entender el reto intelectual que planteó Weber, el estilo en que lo hizo, así como la atmósfera —académica, política, cultural- en que surgió.

Recibido: septiembre de 2014

Revisado: noviembre de 2014

Correspondencia: Centro de Investigación y Docencia Económicas (CIDE)/ Carretera México-Toluca 3655 (Km 16.5)/C.P. 01210/México D.F./correo electrónico: alvaro.morcillo@cide.edu 


\section{Bibliografía}

Breuer, Stefan (2014), "La dominación legítima", en Álvaro Morcillo Laiz y Eduardo Weisz (eds.), Max Weber: una mirada iberoamericana, México, FCE.

Bruun, Hans Henrik (1972), Science, Values and Politics in Max Weber's Methodology, Copenhague, Munksgaard.

Gerth, Hans H. y C. Wright Mills (1946), "Preface", en Max Weber, Hans H. Gerth y C. Wright Mills (eds. y trads.), From Max Weber: Essays in Sociology, Nueva York, Oxford University Press, pp. v-vii.

Hennis, Wilhelm (2015), "Max Weber como educador", en Álvaro Morcillo Laiz y Eduardo Weisz (eds.), Max Weber en Iberoamérica. Nuevas interpretaciones, estudios empíricos y recepción, México, FCE, en prensa.

Hennis, Wilhelm (1987a), "El tema de Max Weber", en Ma. Carmen Iglesias (ed.), Historia y pensamiento: Homenaje a Luis Díez del Corral, Madrid, Eudema, pp. 9-54.

Hennis, Wilhelm (1987b), "A Science of Man - Max Weber and the Political Economy of the German Historical School", en Wolfgang J. Mommsen y Jürgen Osterhammel (eds.), Max Weber and His Contemporaries, Londres, Allen \& Unwin, pp. 25-58.

Hennis, Wilhelm (1983), "El problema central de Max Weber", Revista de Estudios Políticos, núm. 33, pp. 49-100.

Käsler, Dirk (2014), Max Weber. Preusse, Denker, Muttersohn: eine Biographie [Max Weber. Prusiano, pensador, hijo de mamá: una biografía], Munich, Beck. Kaube, Jürgen (2014), Max Weber: Ein Leben zwischen den Epochen [Max Weber: una vida entre épocas], Berlín, Rowohlt.

Mann, Thomas (1979), Tagebücher 1918-1921 [Diarios 1918-1921], edición de Peter Mendelssohn e Inge Jens, Fráncfort del Meno, Fischer.

Mommsen, Wolfgang J. (2014), "Sobre el surgimiento de la obra póstuma de Max Weber 'Economía y sociedad. Sociología”, Estudios Sociológicos, vol. XXXII, núm. 96, pp. 709-766.

Mommsen, Wolfgang J. (2004), Max Weber und die deutsche Politik: 1890-1920, tercera edición, Tubinga, J. C. B. Mohr (Paul Siebeck).

Mommsen, Wolfgang J. (1984), Max Weber and German Politics, 1890-1920, Chicago, The University of Chicago Press.

Monroy, Pedro Andrés Piedras (2008), "Una lectura de Humboldt: Max Weber y la universidad alemana”, Arbor: Ciencia, Pensamiento y Cultura, núm. 731, pp. 481-493.

Morcillo Laiz, Álvaro (2014), "Un vocabulario para la modernidad. Crítica a la interpretación de Max Weber por sus primeros traductores al español", Estudios Sociológicos, vol. XXXII, núm. 96, pp. 767-818.

Morcillo Laiz, Álvaro (2013), "Naturaleza vs. situación vital en Max Weber: dos biografías desiguales”, Revista Española de Investigaciones Sociológicas, núm. 143, julio, pp. 121-127.

Morcillo Laiz, Álvaro (2012), “Aviso a los navegantes. La traducción al español 
de Economía y Sociedad de Max Weber", Estudios Sociológicos, vol. XXX, núm. 90, pp. 609-640.

Pocock, John Greville Agard (1987), "The Concept of a Language and the Métier d'historien: Some Considerations on Practice", en Anthony Pagden (ed.), The Languages of Political Theory in Early-Modern Europe, Cambridge, Cambridge University Press, pp. 19-38.

Radkau, Joachim (2011), Max Weber. La pasión del pensamiento, México, FCE.

Roth, Guenther (2006), "Heidelberg und Montreal. Zur Geschichte des Weberzentenariums", en Karl-Ludwig Ay y Knut Borchardt (eds.), Das Faszinosum Max Weber. Die Geschichte seiner Geltung, Konstanz, UVK, pp. 377-391.

Roth, Guenther (2001), Max Webers deutsch-englische Familiengeschichte 18001950: Mit Briefen und Dokumenten [La historia germano-inglesa de la familia de Max Weber 1800-1950. Con cartas y documentos], Tubinga, J. C. B. Mohr (Paul Siebeck).

Roth, Guenther (1978), "Introduction", en Max Weber, Economy and Society: an Outline of Interpretive Sociology, Guenther Roth y Claus Wittich (eds.), Berkeley, University of California Press, pp. xxvii-cx.

Schluchter, Wolfgang (1989), "Wirtschaft und Gesellschaft - Das Ende eines Mythos" [Economía y sociedad - El final de un mito], en Johannes Weiß (ed.), Max Weber Heute: Erträge und Probleme der Forschung [Max Weber hoy: utilidad y problemas de la investigación], Fráncfort del Meno, Suhrkamp, pp. 55-89.

Schumpeter, Joseph Alois (1944), Teoría del desenvolvimiento económico: una investigación sobre ganancias, capital, crédito, interés y ciclo económico, traducción de Jesús Prados Arrarte, México, FCE.

Schumpeter, Joseph Alois (1912), Theorie der wirtschaftlichen Entwicklung [Teoria del desarrollo económico], Lipsia, Duncker \& Humblot.

Schumpeter, Joseph Alois (1908), Das Wesen und der Hauptinhalt der theoretischen Nationalökonomie [La esencia y el contenido básico de la economía política], Lipsia, Duncker \& Humblot.

Stammer, Otto (1971), Max Weber and Sociology Today, Nueva York, Harper \& Row.

Stammer, Otto (1965), Max Weber und die Soziologie Heute [Max Weber y la sociología hoy], Tubinga, J. C. B. Mohr (Paul Siebeck).

Swedberg, Richard (2007), "Max Weber's Interpretive Economic Sociology", American Behavioral Scientist, vol. 50, núm. 8, pp. 1035-1055.

Swedberg, Richard (2002), "What Can New Economic Sociology Learn from the Historical School, Especially Max Weber", en Heino Heinrich Nau y Bertram Schefold (eds.), The Historicity of Economics: Continuities and Discontinuities of Historical Thought in $19^{\text {th }}$ and $20^{\text {th }}$ Century Economics, Berlín, Springer, pp. 176-193.

Swedberg, Richard (2001), "Schumpeter, Joseph A (1883-1950)", en Paul B. Baltes y Neil J. Smelser (eds.), International Encyclopedia of the Social \& Behavioral Sciences, Londres, Elsevier, pp. 13598-13603.

Swedberg, Richard (1998), Max Weber and the Idea of Economic Sociology, Princeton, Princeton University Press. 
Tenbruck, Friedrich H. (1977), "Abschied von Wirtschaft und Gesellschaft" [Despedida de Economía y sociedad], Zeitschrift für die gesamte Staatswissenschaft, núm. 133, pp. 703-736.

Tribe, Keith (2015), “¿Qué es la Sozialökonomik?”, en Álvaro Morcillo Laiz y Eduardo Weisz (eds.), Max Weber en Iberoamérica. Nuevas interpretaciones, estudios empiricos y recepción, México, FCE, en prensa.

Tribe, Keith (2012), "Max Weber: the Works", Economy and Society, vol. 41, núm. 2, pp. 282-298.

Tribe, Keith (1995), Strategies of Economic Order: German Economic Discourse, 1750-1950, Cambridge, Cambridge University Press.

Weber, Max (2015), Hochschulwesen und Wissenschaftspolitik. Schriften und Reden 1895-1920 [Universidad y política científica. Escritos y conferencias], edición de M. Rainer Lepsius, Max Weber Gesamtausgabe I/13, Tubinga, J. C. B. Mohr (Paul Siebeck), en preparación.

Weber, Max (2009), Allgemeine ("theoretische") Nationalökonomie. Vorlesungen 1894-1898 [Economía política general ("teórica") Lecciones 1894-1898], Gesamtausgabe, edición de Wolfgang J. Mommsen, Cristof Judenau, Heino H. Nau, Klaus Scharfen y Marcus Tiefel, III/1, Tubinga, J. C. B. Mohr (Paul Siebeck).

Weber, Max (2003), Briefe: 1913-1914, Max Weber Gesamtausgabe, edición de Mario Rainer Lepsius, Horst Baier, Rudhard Birgit y Manfred Schön, II/8. Tubinga, J. C. B. Mohr (Paul Siebeck).

Weber, Max (1991) [1895], "El Estado Nacional y la política económica. Discurso de toma de posesión de la cátedra", en M. Weber, Escritos políticos, traducción de Joaquín Abellán, Madrid, Alianza, pp. 66-100.

Weber, Max (1988) [1895], "Der Nationalstaat und die Volkswirtschaftspolitik. Akademische Antrittsrede", en Johannes Winckelmann (ed.), Gesammelte Politische Schriften, quinta edición, Tubinga, J. C. B. Mohr (Paul Siebeck), pp. 1-26.

Weber, Max (1985a) [1913], "Über einige Kategorien der verstehenden Soziologie" ["Sobre algunas categorías de la sociología comprensiva"], en Johannes Winckelmann (ed.), Gesammelte Aufsätze zur Wissenschaftslehre, Tubinga, J. C. B. Mohr (Paul Siebeck), pp. 427-474.

Weber, Max (1985b) [1917], "Der Sinn der 'Wertfreiheit' der soziologischen und ökonomischen Wissenschaften", en Johannes Winckelmann (ed.), Gesammelte Aufsätze zur Wissenschaftslehre, Tubinga, J. C. B. Mohr (Paul Siebeck), pp. 489540 .

Weber, Max (1985c) [1904], "Die «Objektivität» sozialwissenschaftlicher und sozialpolitischer Erkenntnis", en Johannes Winckelmann (ed.), Gesammelte Aufsätze Zur Wissenschaftslehre, Tubinga, J. C. B. Mohr (Paul Siebeck), pp. 146-214.

Weber, Max (1974), Max Weber on Universities: the Power of the State and the Dignity of the Academic Calling in Imperial Germany, edición de Edward Shils, Chicago, University of Chicago Press.

Weber, Max (1973a) [1913], "Sobre algunas categorías de la sociología comprensiva", en M. Weber, Ensayos sobre metodología sociológica, traducción de José Luis Etcheverry, Buenos Aires, Amorrortu, pp. 175-221. 
Weber, Max (1973b) [1917], "El sentido de la 'neutralidad valorativa' de las ciencias sociológicas y económicas”, en M. Weber, Ensayos sobre metodología sociológica, traducción de José Luis Etcheverry, Buenos Aires, Amorrortu, pp. 222-269. Weber, Max (1973c) [1904], “La ‘objetividad' cognoscitiva de la ciencia social y de la política social”, en M. Weber, Ensayos sobre metodología sociológica, traducción de José Luis Etcheverry, Buenos Aires, Amorrortu, pp. 39-101.

Weber, Max (1944) [1922], Economia y sociedad. I. Teoría de la organización social, traducción de José Medina Echavarría, IV vols., México, FCE.

Weber, Max (1922), Wirtschaft und Gesellschaft [Economía y sociedad], Tubinga, J. C. B. Mohr (Paul Siebeck).

Zaret, David (1980), "From Weber to Parsons and Schutz: the Eclipse of History in Modern Social Theory", American Journal of Sociology, vol. 85, núm. 5, pp. 1180-1201.

\section{Acerca del autor}

Álvaro Morcillo Laiz es doctor por la Universidad Humboldt de Berlín. Actualmente se desempeña como profesor-investigador titular de la División de Estudios Internacionales del Centro de Investigación y Docencia Económicas (CIDE). Sus intereses de investigación son la sociología de las ideas y la historia de las ciencias sociales, así como la política comparada y la integración europea. Entre sus obras recientes están "La dominación filantrópica. La Fundación Rockefeller, El Colegio de México, el Instituto di Tella y las ciencias sociales en español (1938-1973)", en Álvaro Morcillo Laiz y Eduardo Weisz (editores), Max Weber en Iberoamérica. Nuevas interpretaciones, estudios empíricos y recepción, México, FCE, 2015; así como "Un vocabulario para la modernidad. Crítica a la interpretación de Max Weber por sus primeros traductores al español", Estudios Sociológicos, vol. XXXII, núm. 96, 2014, pp. 767-818. 
